\title{
CCM PILOT STUDY OVERVIEW: GEOMETRICAL MEASUREMENT OF THE ROCKWELL DIAMOND INDENTER
}

\author{
R. R. Machado ${ }^{1}$, S. Low ${ }^{2}$, A. Germak ${ }^{3}$ \\ ${ }^{1}$ INMETRO, Duque de Caxias, Brazil rrmachado@inmetro.gov.br \\ ${ }^{2}$ NIST, Gaithersburg, USA, samuel.low@nist.gov \\ ${ }^{3}$ INRiM, Torino, Italy, a.germak@inrim.it
}

\begin{abstract}
:
This paper describes an overview of the capability of the NMIs that participated on the CCM Pilot Study measurement systems, conducted by the CIPM/CCM/Working Group on Hardness, to characterize the Rockwell hardness diamond indenter geometry, by measuring the included cone angle, the straightness of the generatrix, the spherical tip radius, the deviation of the local radius and the tilt angle.

Nine NMIs took part in this study: INMETRO (Brazil); INRiM (Italy); KRISS (South Korea); NIM/PR (China); NIMT (Thailand); NIST (USA); PTB (Germany); TUBITAK UME (Turkey); VNIIFTRI (Russia), where INMETRO (Brazil) served as pilot laboratory.
\end{abstract}

Keywords: hardness, diamond indenter, geometry indenter, pilot study, CIPM/CCM/WGH

\section{INTRODUCTION}

International hardness measurement equivalence between the National Metrology Institutes (NMIs) is the role of the Consultative Committee for Mass and Related Quantities (CCM) Working Group on Hardness (WGH) within the International Committee of Weights and Measures (CIPM) [1]. There have been numerous discussions among the members of the CCM-WGH concerning the difficulty in obtaining calibration-grade Rockwell hardness diamond indenters having the correct shape within geometric tolerances as specified in international standards. There are two main reasons for this difficulty. The primary reason is that the crystallographic structure of diamond makes it extremely difficult to grind and polish to the required Rockwell indenter shape. The Rockwell indenter shape is a cone having a $120^{\circ}$ included angle and a spherical tip radius of $0.2 \mathrm{~mm}$. Secondly, the accurate measurement of a Rockwell indenter's geometrical parameters is a difficult and often time-consuming process due to the conical and spherical indenter geometries and the optical transparency and high reflectivity of the polished diamond.
Therefore, the CCM-WGH agreed to carry out a Pilot Study (PS) on the measurement of Rockwell hardness diamond indenter geometries in which the laboratories of National Metrology Institutes (NMIs) should participate. INMETRO (Brazil) was designated as the Pilot Laboratory. The Pilot Study was conducted from December 2011 until January 2015 , with all but the repeat measurements of the Pilot Laboratory completed by March 2013.

\section{PRINCIPLE OF THE PILOT STUDY}

The purpose of this CCM-PS was to document how the NMIs are measuring, analyzing and reporting results of their indenter geometry calibrations. This study was intended to reveal current NMI calibration capabilities and any measurement issues, before conducting a CCM - Key Comparison that will establish measurement equivalence between the NMIs engaged in the metrological characterization of diamond indenters.

\subsection{Test artifacts (measurand)}

Three Rockwell hardness diamond indenters designated as indenters 104, 122 and 130 (as engraved on them) were measured for specific geometry parameters by the participating laboratories.

These three indenters were chosen for the Pilot Study from an inventory of indenters previously measured and provided by NIST/USA. They were circulated among the laboratories with the following characteristics: one indenter was believed to have a near-nominal shape while the other two were less accurate but within permissible limits defined by ISO 6508-3 [2]. Figure 1 gives the approximate overall dimensions of them indenters.

\subsection{Format of the comparison}

The participating laboratories conducted dimensional measurements of specified geometric parameters on each of the three Rockwell diamond indenters using their usual procedures for qualifying them, and according to the capacity of their measurement systems. Those laboratories that did not 
have their own procedures to qualify Rockwell diamond indenters were instructed to follow the requirements defined in ISO 6508 Part 3 [2]. Deviations from the procedures established in this standard were to be reported.

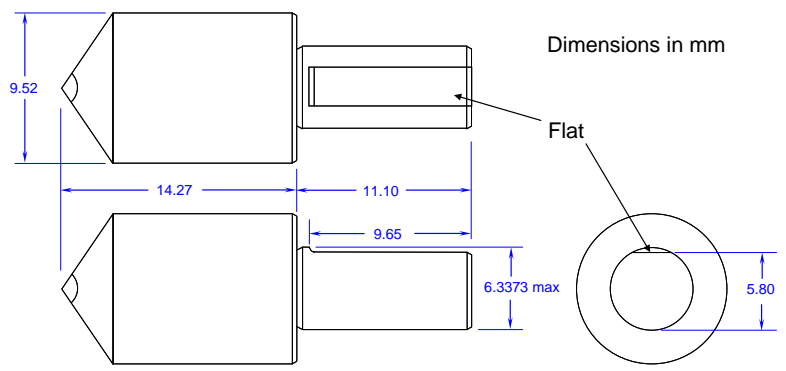

Figure 1: Approximate dimensions of the indenters

The dimensional measurements were intended to illustrate measurement biases among NMIs and measurement differences between different types of measuring instruments. Figure 2 shows the geometry of a Rockwell diamond indenter, according to the following parameters and limits defined in the ISO 6508-3 [2].

- Included cone angle of the diamond $(\theta)$.

Limits from the mean value: $(120 \pm 0.1)^{\circ}$.

- Radius of the spherical tip of the diamond $(r)$.

Limits from the mean value: $(0.200 \pm 0.005) \mathrm{mm}$.

- Profile deviation of the local radius from the least square mean radius value.

Limit: $\max 0.002 \mathrm{~mm}$.

- Straightness of the generatrix of the diamond cone, adjacent to the blend of the spherical and conical parts.

Limit from the mean deviation: $\max 0.0005 \mathrm{~mm}$ over a minimum length of $0.4 \mathrm{~mm}$.

- Angle between the axis of the diamond cone and the axis normal to the seating surface.

Limit: $\max 0.3^{\circ}$.

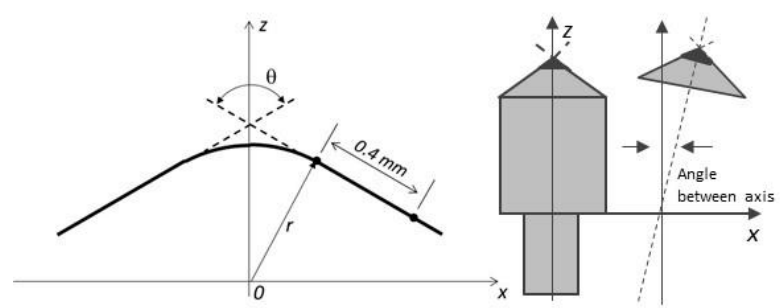

Figure 2: Illustration of the geometries of the Rockwell diamond indenter

\subsection{Indenter calibration systems}

A Rockwell diamond indenter calibration system should be capable of making the dimensional measurements specified in ISO 6508-3 [2].

For measurement systems that make axial-plane measurements, if possible, the diamond shape measurements should be made at the 8 section locations as illustrated in Figure 3. Measurement Position 1 is in the axial plane of the indenter that is parallel with the flat on the indenter shaft. Position 2 is in the axial plane rotated $45^{\circ}$ clockwise around the indenter axis from Position 1 as the indenter tip points towards the operator. The remaining 6 measurements should be made similarly at $45^{\circ}$ rotations.

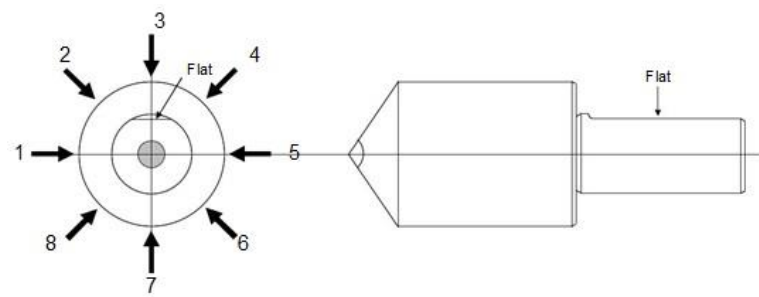

Figure 3: Illustration of the measurement sections of the Rockwell diamond indenter. The figure on the left is oriented with diamond tip pointing towards to the operator [3]

\section{METHOD OF ANALYSIS}

Two methods of analysis were used to determine the dispersion of all data points obtained by the Labs. The first one was the Weighted mean method, which is one of the most used methods in comparisons, based on the reproducibility between the Labs (standard deviation of the mean). The second one was based on the NIST Consensus Builder (NICOB), which is consistent with the combination of measurement results obtained independently by different laboratories or measurement methods [4, 5].

\subsection{Weighted mean method}

One of the methods used to analyze the results of the comparison and determine the dispersion of the data points obtained by the Labs was the weighted mean method, Equation (1).

$$
\begin{array}{ll}
\bar{w}= & \frac{\sum_{i}^{n} x_{i} \times w_{i}}{\sum_{i}^{n} w_{i}}, \text { where } \\
\bar{w} & \text { weighted mean } \\
x_{i} \quad \begin{array}{l}
\text { measured values provided by the NMI } \\
\text { participant }
\end{array} \\
w_{i} \quad \text { weight } \\
w_{i}=\frac{1}{u_{i}^{2}}, \text { where } \\
u_{i} \quad \text { standard uncertainty of } x_{i} .
\end{array}
$$

From the above method of analysis, the normalized errors from the weighted mean (En.w), Equation (3), were calculated, and the respective graphics were plotted (clause 4 ).

$$
\text { En. } w=\frac{\left(x_{i}-\bar{w}\right)}{\sqrt{{U_{i}}^{2}+U_{r e f}^{2}}}, \text { where }
$$

En.w normalized error from the weighted mean

$x_{i} \quad$ measured mean values provided by the NMI participants

$\bar{W} \quad$ weighted mean value

$U_{i} \quad$ expanded uncertainty provided by the NMI participants $(k=2)$

$U_{\text {ref }}$ reference value of the expanded uncertainty from weighted mean. 


\subsection{NICOB method}

The other method used to analyze the results of the comparison, and to determine the dispersion of the obtained data points by the labs was the method based on the NIST Consensus Builder (NICOB) methodology. This methodology is consistent with the combination of measurement results obtained independently by different laboratories or measurement methods $[4,5]$.

Among the three procedures available in the NICOB method, the Dersimonian-Laird (DL) seemed to be the most suitable for this case, and it was the procedure applied to this analysis. This procedure uses Equation (4) as a weighted mean.

$$
\begin{aligned}
& \overline{\boldsymbol{w}}_{\boldsymbol{D} \boldsymbol{L}}=\frac{\sum_{i}^{n} \boldsymbol{x}_{i} \times \boldsymbol{w}_{i . D L}}{\sum_{i}^{n} \boldsymbol{w}_{i . D L}}, \text { where } \\
& \overline{\boldsymbol{w}}_{\boldsymbol{D L}} \text { Dersimonian-Laird weighted mean } \\
& \boldsymbol{x}_{\boldsymbol{i}} \quad \text { measured values provided by the NMI } \\
& \text { participant } \\
& \boldsymbol{w}_{\boldsymbol{i} . \boldsymbol{D} \boldsymbol{L}} \text { Dersimonian-Laird weight } \\
& \boldsymbol{w}_{i . D L}=\frac{1}{\boldsymbol{u}_{i}^{2}+\tau^{2}} \text {, where } \\
& \boldsymbol{u}_{\boldsymbol{i}}^{2} \quad \text { uncertainty associated with the value } \\
& \text { measured by the NMIs } \\
& \tau^{2} \text { indication of the heterogeneity of the } \\
& \text { measured results }
\end{aligned}
$$

From the above method of analysis, the normalized errors from the Dersimonian-Laird weighted mean (En.DL), Equation (6), were then calculated, and the respective graphics were plotted (clause 4).

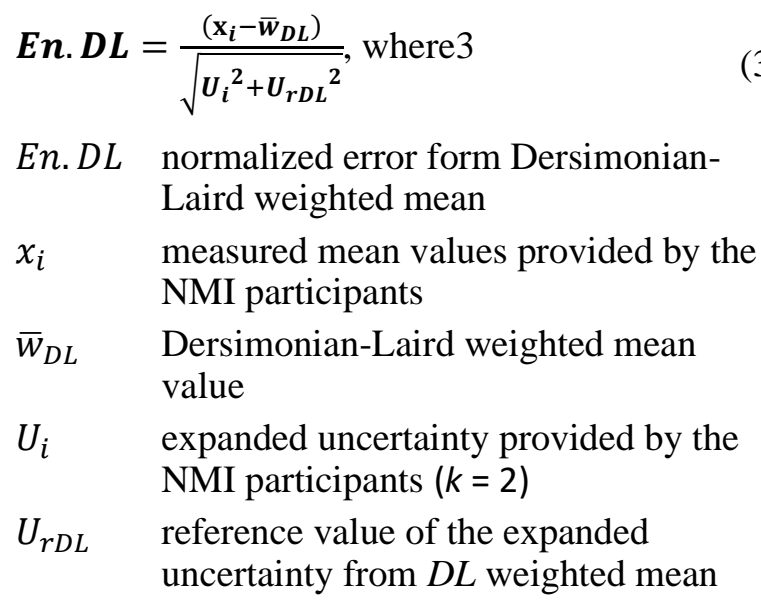

\section{RESULTS}

Some NMIs provided more than one measurement result for the same parameter based on measuring different areas or windows of the indenter surface. Consequently, for each window, different values were obtained for the same indenter. The windows considered in this report, were those around the window values described by John Song et al. [6]. They said that for an ideally shaped Rockwell indenter, as specified in the standards [7] and [2], i.e., $200 \mu \mathrm{m}$ tip radius blending with a $120^{\circ}$ cone angle in a true tangential manner, the window sizes must be $\pm 100 \mu \mathrm{m}$ for the tip radius calibration; and $\pm(100$ to 450$) \mu \mathrm{m}$ along the $\mathrm{x}$-axis for the left and right contributions to the cone angle calibration, and the sizes must be specified in the data analysis.

The choice of the measurement window is especially important for calculation of least squares radius and profile deviation in the center and cone flank straightness in the left and right. Furthermore, the blend area of the tip radius and cone angle must be accounted for when choosing window positions. Beyond these considerations, John Song et al. [6], said that the blend point can change depending on the actual cone angle and tip radius. In light of these deviations, both the ASTM and ISO standards specify that the straightness of the cone flank is measured "adjacent to the blend" which leaves some flexibility in the choice of the size and position of the windows on the flanks.

\subsection{Results of the Weighted mean $(\bar{w})$, Dersimonian-Laird weighted mean $\left(\bar{w}_{D L}\right)$ and their expanded uncertainties}

In the coming clauses, we present the results of the mean values measured by the NMI participants and their respective measurement uncertainties for each parameter. For convenience, the graphics from Figure 4 to Figure 8 are showing only the results related to the weighted mean.

\subsubsection{Included cone angle}

The NMIs measurement results related to the geometric parameter "included cone angle" are shown in Figure 4 for the three indenters. In general, it can be seen in this figure good agreement between the NMI results.

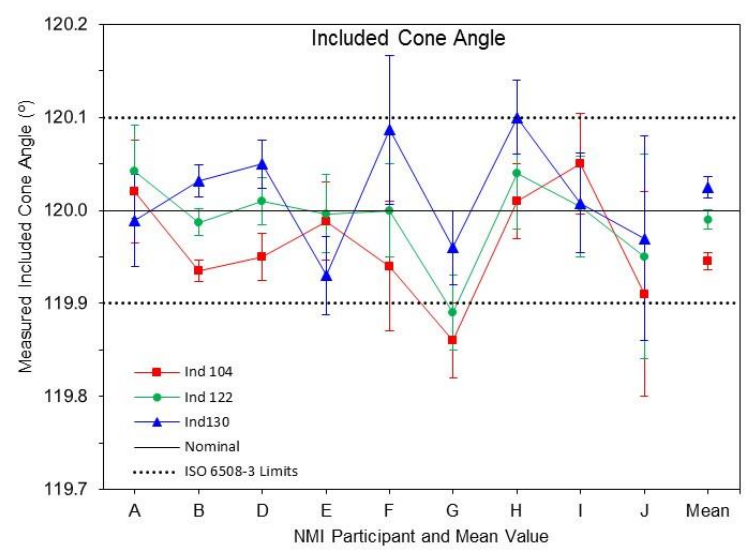

Figure 4: The weighted mean values and the respective uncertainties (error bars) of the included cone angle for all indenters and the maximum limits specified by ISO 65083

The only exceptions were due to the larger uncertainty provided by NMIs " $F$ ' and " $J$ ", according to the error bars. We note that NMI "G" would fail this parameter for indenters 104 and 122 while all other NMIs would pass them. 


\subsubsection{Radius of the spherical tip}

Figure 5 presents the data related to the geometric parameter "radius of the spherical tip". It is possible to see a very good dispersion among the obtained results for the three indenters, measured by all NMI participants. However, for indenters 122 and 130, some NMIs would have passed the radius geometry of the two indenters while others would have failed their compliance with ISO 6508-3. Also, NMI "E" reported a larger uncertainty than the other NMIs, mainly when measuring the indenters 104 and 130.

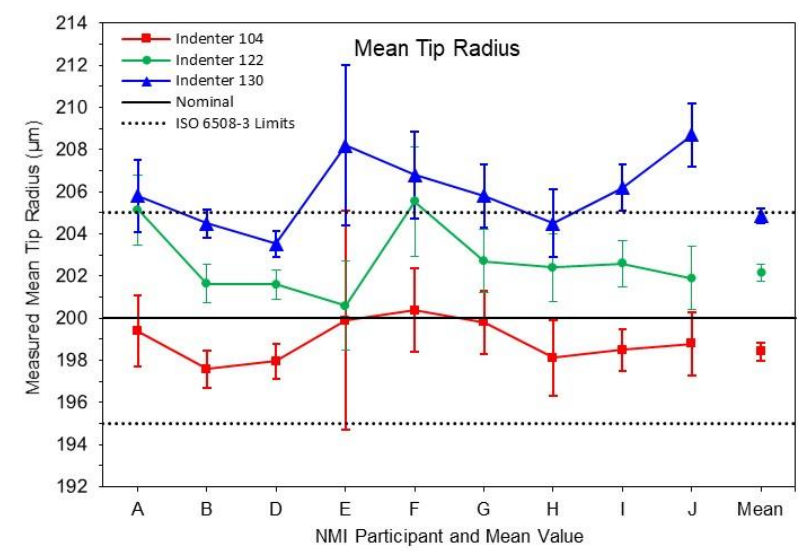

Figure 5: The weighted mean values and the respective uncertainties (error bars) of the radius of the spherical tip for all indenters

\subsubsection{Maximum profile deviation of the radius from the true radius}

Figure 6 presents graphics related to the geometric parameter "max profile deviation" for the indenters 104, 122 and 130. It is noted that only five NMIs reported this measurement indicating an expansion of measurement capabilities may be needed at some NMIs.

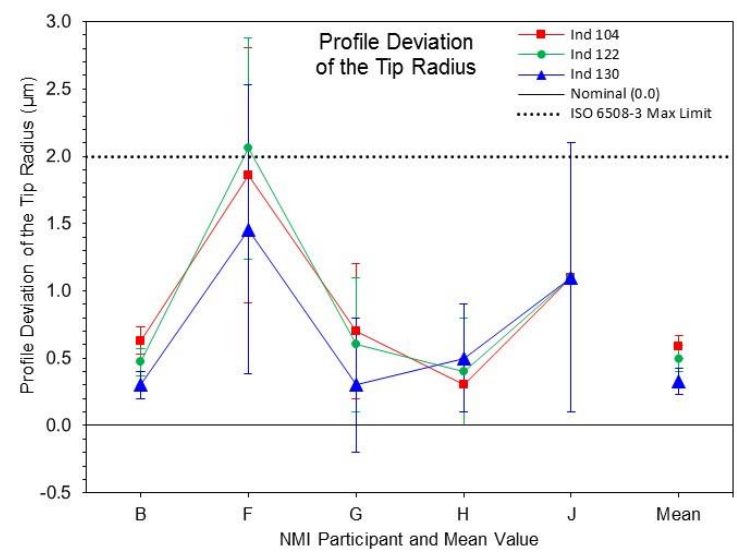

Figure 6: The weighted mean values and the respective uncertainties (error bars) of the max profile deviation for all indenters

Two of the NMI's results varied from the other three with one NMI's results for one indenter exceeding the ISO 6508-3 maximum limit, possibly due to measuring different window sizes and/or higher levels of measurement uncertainty.

\subsubsection{Straightness of the diamond cone generatrix}

Figure 7 presents graphics related to the geometric parameter "straightness of the generatrix" for all three measurands. It is possible to notice in this figure there is a large variation in the reported measurement uncertainties, however these uncertainties do not explain the measurement differences. Furthermore, almost half of the NMIs would have failed the three indenters for exceeding the limit specified by ISO 6508-3 for this parameter. A contribution to these measurement differences may be due to choosing a measurement window that includes part of the tip-radius curvature in the blend area which would negatively affect the measurement. It is also noted that only seven NMIs reported this measurement, again indicating an expansion of measurement capabilities may be needed at some NMIs.

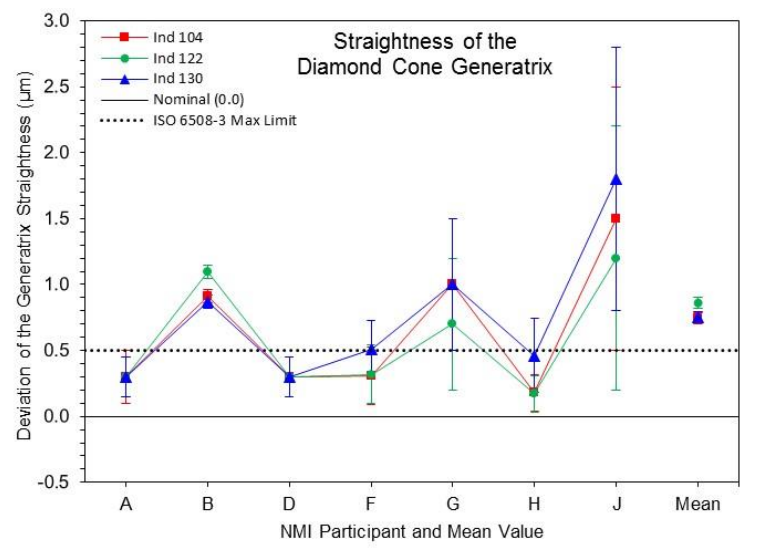

Figure 7: The weighted mean values and the respective uncertainties (error bars) of the straightness of the generatrix for all indenters

\subsubsection{Angle between the axis of the diamond cone} and the axis normal to the seating surface

Figure 8 presents graphics related to the geometric parameter "angle between axes" for the indenters 104, 122 and 130. It is possible to see in these graphics that, with the exception of NMI "G", which stayed very far beyond the mean values, all other NMIs stayed around the average values.

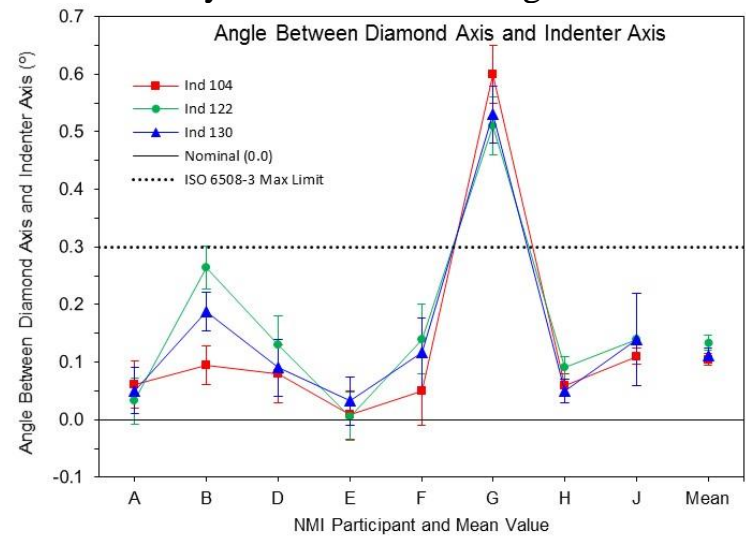

Figure 8: The weighted mean values and the respective uncertainties (error bars) of the angle between axes for all indenters 


\subsection{Results of the normalized errors $\left(E_{\mathbf{n}}\right)$}

This section reports the normalized errors calculated for the measurement results of the geometric parameters of the three indenters measured by the NMI participants of this Pilot Study.

\subsubsection{Included cone angle}

The behavior of $E_{\mathrm{n}}$, calculated for all NMIs related to the parameter "included cone angle", is described in Figure 9. It is possible to see in this figure that the influence caused by the two different methods of analysis were small. However, almost half of NMI's measurements did not agree well with the mean values for one or more of the indenters. It is curious that, for some NMIs, the measurement of this parameter on one indenter is outside the agreement with the other NMIs while the same measurements on the other two indenters are in better agreement. This could possibly be explained by different blend locations on the indenters and choices of window sizes.

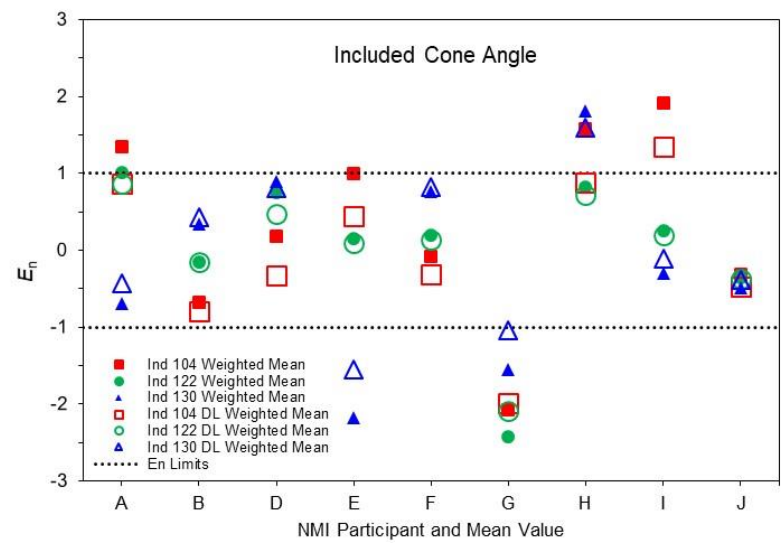

Figure 9: $E_{\mathrm{n}}$ graphics related to the weighted mean and DL mean values of the included cone angle for all indenters

\subsubsection{Radius of the spherical tip}

Figure 10 is related to $E_{\mathrm{n}}$ calculated for the parameter radius of the spherical tip. In this figure, it is possible to see that the results of all NMIs are reasonably consistent no matter the method of analysis considered.

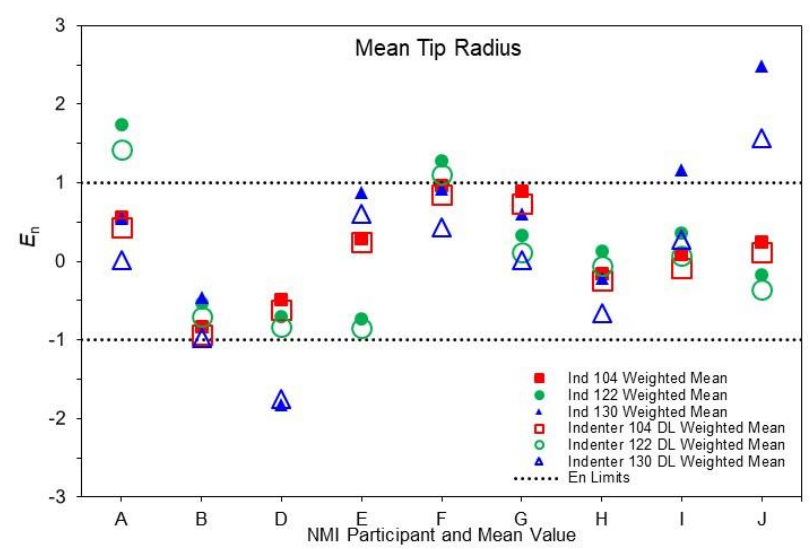

Figure 10: $E_{\mathrm{n}}$ graphics of the weighted mean and DL mean values of the radius tip for all indenters
As with the measurement of cone angle, several NMI's measurements of this parameter on one indenter did not agree well with the mean measurement values while their measurements of the other indenters were consistent with the other NMIs. Again, this could possibly be explained by different blend locations on the indenters and choices of measurement windows.

\subsubsection{Maximum profile deviation of the radius from the true radius}

The following Figure 11, related to the parameter max profile deviation of the radius shows the behavior of $E_{\mathrm{n}}$, calculated for the five NMIs that provided the results of this parameter. It is possible to see in these graphics that the NMI "F" came outside the $E_{\mathrm{n}}$ tolerance for almost all indenters measured, with the exception for the measurement of the indenter 130 , but only when the DL weighted mean method of analysis is considered.

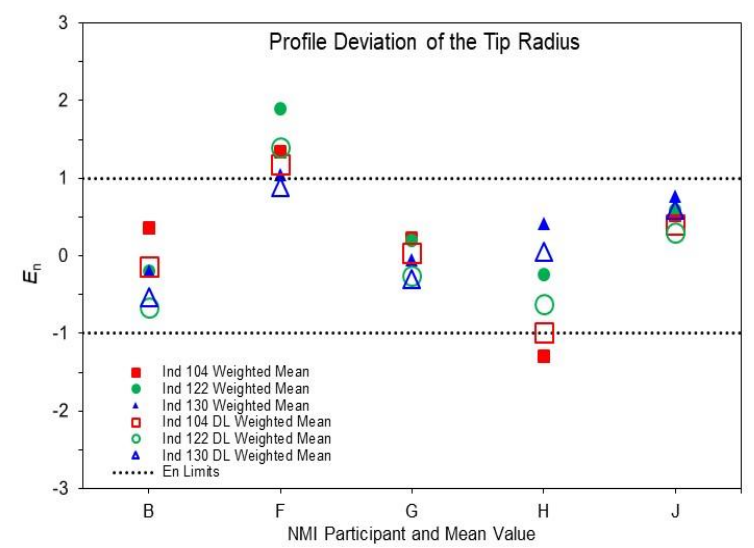

Figure 11: $E_{\mathrm{n}}$ graphics related to the weighted mean and DL mean values of the profile deviation for all indenters

\subsubsection{Straightness of the diamond cone generatrix}

In Figure 12, which is related to the parameter "straightness of the generatrix of the diamond cone", it is easy to see the dependence of $E_{\mathrm{n}}$ behavior calculated for all NMIs on the two different methods used for the analyses.

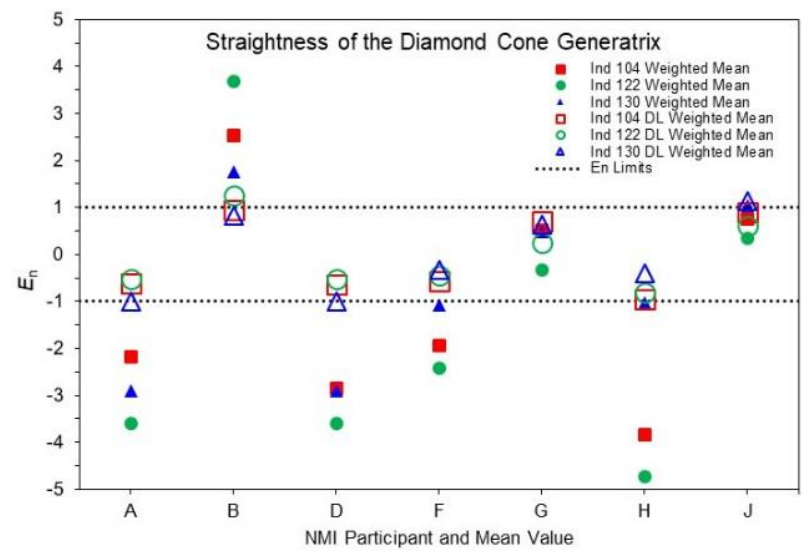

Figure 12: $E_{\mathrm{n}}$ graphics related to the weighted mean and DL mean values of the straightness of the generatrix for all indenters 
The DL analysis provided significantly improved results for the NMIs "A", "B", "D", " $F$ " and " $H$ " that had reported smaller uncertainties, and all measurements analyzed by the DL method were within the $E_{\mathrm{n}}$ limits except for only two cases. The DL analysis is clearly an improvement over the weighted-mean analysis for these NMI results, and it may provide a better representation of equivalence when there is a large variation in measurement uncertainties.

\subsubsection{Angle between the axis of the diamond cone and the axis normal to the seating surface}

Figure 13 is related to $E_{\mathrm{n}}$ calculated for the parameter "angle between axes." As with the parameter, "straightness of the generatrix of the diamond cone", for many of the NMIs the DL analysis provided significantly improved results compared with the weighted-mean method. However, many NMI measurements continued to fall outside the $E_{\mathrm{n}}$ limits for some or all indenters.

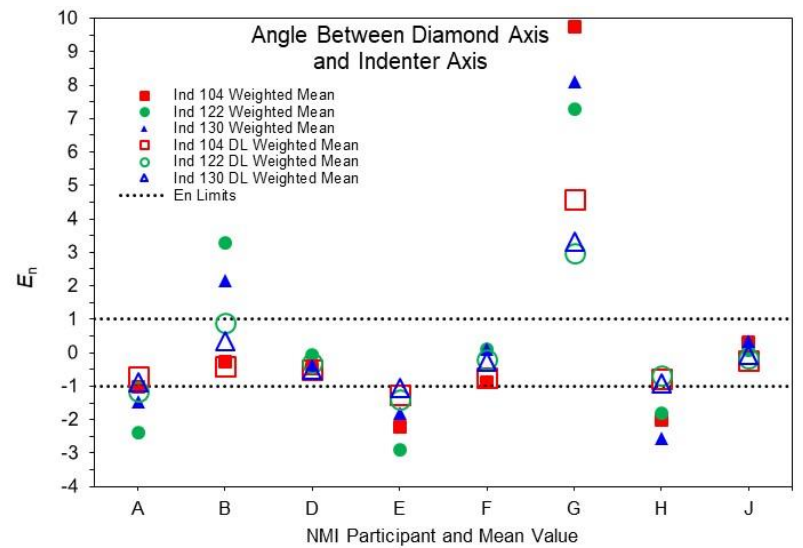

Figure 13: $E_{\mathrm{n}}$ graphics related to the weighted mean and DL mean values of the angle between axes for all indenters

\section{DISCUSSIONS}

This comparison included nine participating labs from National Metrology Institutes, of Europe, Asia and the Americas, where they had to measure the same geometric parameters of three indenters. However, no reference value and thus no uncertainty of a reference value were assigned. Each Lab obtained an average value of the same number of measurements, for each parameter, and reported the mean values with their respective measurement uncertainties.

Considering the measurement results and the plotted graphics (clause 4), this Pilot Study indicated several issues that the participating NMIs should address in order to fully characterize a Rockwell hardness diamond indenter, and the CCM-WGH should address in developing the test protocol for a future key comparison. These include:

- Some NMIs have difficulty making measurements of some geometric parameters. This is evident from the examples of NMIs having very different results than the other NMIs.

- Some NMIs need to expand their capabilities to measure all pertinent parameters. There were several NMIs that did not report certain parameter measurement results.

- Measurement uncertainties are being underestimated and/or the same quantity (i.e., area) is not being measured. There were many examples where the reported uncertainties could not account for the measurement differences. This may not have resulted solely from poor estimations of uncertainties but may also be the result of not measuring the same geometric area.

- Improved definitions or instructions of what to measure are needed. The result of NMIs using different window sizes may have been the primary cause of differences in the measurements related to the tip radius and cone angle, particularly when the radius/cone blend area was included in the measurements.

- The NICOB DL data analysis may provide improved evidence of measurement equivalence as compared to the Weighted-Mean analysis.

The consequence of not addressing these issues is that some NMIs may pass an indenter parameter's incorrect geometry as specified by the consensus test methods of ISO and ASTM, while other NMIs may properly fail it, and vice versa. This has the potential of increasing differences between national Rockwell hardness standards.

\section{REFERENCES}

[1] https://www.bipm.org/en/committees/cc/wg/ccmwgh.html

[2] ISO 6508-3:2005 Metallic materials - Rockwell hardness test - Part 3: Calibration of reference blocks (scales A, B, C, D, E, F, G, H, K, N, T), International Organisation for Standardisation, Geneva, Switzerland.

[3] J. F. Song, F. F. Rudder, Jr., T. V. Vorburger, and J. H. Smith. Microform Calibration Uncertainties of Rockwell Diamond Indenters. J. Res. Natl. Inst. Stand. Technol. 1995 Sep-Oct; 100(5): 543-561.

[4] NIST Consensus Builder User's Manual. Amanda Koepke, Thomas Lafarge, Antonio Possolo, Blaza Toman. Statistical Engineering Division Information Technology Laboratory. National Institute of Standards and Technology, Gaithersburg, MD, 2017.

[5] Amanda Koepke et al. 2017 Metrologia 54 S34. https://doi.org/10.1088/1681-7575/aa6c0e

[6] J. Song, S. Low, A. Zheng and P. Gu. Geometrical measurements of NIST SRM Rockwell hardness diamond indenters. In: IMEKO 2010 TC3, TC5 and TC22 Conferences Metrology in Modern Context. November 22-25, 2010, Pattaya, Chonburi, Thailand.

[7] ASTM E18-20, Standard Test Methods for Rockwell Hardness of Metallic Materials, ASTM International, West Conshohocken, PA, 2020, www.astm.org 